\title{
A method for estimating the turbulent kinetic energy dissipation rate from a vertically pointing Doppler lidar, and independent evaluation from balloon- borne in situ measurements
}

Article

Published Version

O'Connor, E. J., Illingworth, A. J., Brooks, I. M., Westbrook, C. D., Hogan, R. J., Davies, F. and Brooks, B. J. (2010) A method for estimating the turbulent kinetic energy dissipation rate from a vertically pointing Doppler lidar, and independent evaluation from balloon-borne in situ measurements. Journal of Atmospheric and Oceanic Technology, 27 (10). pp. 1652-1664. ISSN 1520-0426 doi:

https://doi.org/10.1175/2010JTECHA1455.1 Available at https://centaur.reading.ac.uk/16256/

It is advisable to refer to the publisher's version if you intend to cite from the work. See Guidance on citing.

To link to this article DOI: http://dx.doi.org/10.1175/2010JTECHA1455.1

Publisher: American Meteorological Society

Publisher statement: (C) Copyright October 2010 American Meteorological Society (AMS). Permission to use figures, tables, and brief excerpts from this work in scientific and educational works is hereby granted provided that the source is 
acknowledged. Any use of material in this work that is determined to be "fair use" under Section 107 of the U.S. Copyright Act September 2010 or that satisfies the conditions specified in Section 108 of the U.S. Copyright Act (17 USC §108, as revised by P.L. 94-553) does not require the AMS's permission. Republication, systematic reproduction, posting in electronic form, such as on a web site or in a searchable database, or other uses of this material, except as exempted by the above statement, requires written permission or a license from the AMS.

Additional details are provided in the AMS Copyright Policy, available on the AMS Web site located at (http://www.ametsoc.org/) or from the AMS at 617-227-2425 or copyright@ametsoc.org.

All outputs in CentAUR are protected by Intellectual Property Rights law, including copyright law. Copyright and IPR is retained by the creators or other copyright holders. Terms and conditions for use of this material are defined in the End User Agreement.

\section{www.reading.ac.uk/centaur}

\section{CentAUR}

Central Archive at the University of Reading

Reading's research outputs online 


\title{
A Method for Estimating the Turbulent Kinetic Energy Dissipation Rate from a Vertically Pointing Doppler Lidar, and Independent Evaluation from Balloon-Borne In Situ Measurements
}

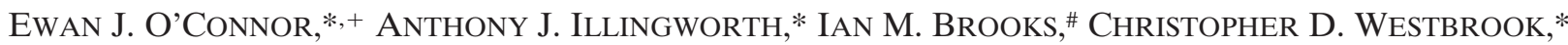 \\ ROBIN J. HOGAN,* FAY DAVIES, ${ }^{\circledR}$ AND BARBARA J. BROOKS\# \\ * Department of Meteorology, University of Reading, Reading, United Kingdom \\ ${ }^{+}$Finnish Meteorological Institute, Helsinki, Finland \\ \# University of Leeds, Leeds, United Kingdom \\ ${ }^{\circledR}$ School of Environment and Life Sciences, University of Salford, Salford, United Kingdom
}

(Manuscript received 9 February 2010, in final form 27 May 2010)

\begin{abstract}
A method of estimating dissipation rates from a vertically pointing Doppler lidar with high temporal and spatial resolution has been evaluated by comparison with independent measurements derived from a balloonborne sonic anemometer. This method utilizes the variance of the mean Doppler velocity from a number of sequential samples and requires an estimate of the horizontal wind speed. The noise contribution to the variance can be estimated from the observed signal-to-noise ratio and removed where appropriate. The relative size of the noise variance to the observed variance provides a measure of the confidence in the retrieval. Comparison with in situ dissipation rates derived from the balloon-borne sonic anemometer reveal that this particular Doppler lidar is capable of retrieving dissipation rates over a range of at least three orders of magnitude.

This method is most suitable for retrieval of dissipation rates within the convective well-mixed boundary layer where the scales of motion that the Doppler lidar probes remain well within the inertial subrange. Caution must be applied when estimating dissipation rates in more quiescent conditions. For the particular Doppler lidar described here, the selection of suitably short integration times will permit this method to be applicable in such situations but at the expense of accuracy in the Doppler velocity estimates. The two case studies presented here suggest that, with profiles every $4 \mathrm{~s}$, reliable estimates of $\epsilon$ can be derived to within at least an order of magnitude throughout almost all of the lowest $2 \mathrm{~km}$ and, in the convective boundary layer, to within $50 \%$. Increasing the integration time for individual profiles to $30 \mathrm{~s}$ can improve the accuracy substantially but potentially confines retrievals to within the convective boundary layer. Therefore, optimization of certain instrument parameters may be required for specific implementations.
\end{abstract}

\section{Introduction}

Turbulent properties of the boundary layer can be measured by aircraft (e.g., Fairall et al. 1980) and vertical profiles of the turbulent kinetic energy dissipation rate have been obtained from balloon-borne turbulence probes in the convective boundary layer (Caughey and Palmer 1979), nocturnal boundary layer (Caughey et al. 1979), and cloudy boundary layers (Hignett 1991; Siebert

Corresponding author address: Ewan J. O'Connor, Dept. of Meteorology, University of Reading, Earley Gate, P.O. Box 243, Reading RG6 6BB, United Kingdom.

E-mail: e.j.oconnor@reading.ac.uk et al. 2003). Such in situ observations, however, are necessarily restricted both spatially and temporally.

Applications such as investigating the role of turbulence in new aerosol particle formation (Wehner et al. 2010), or cloud microphysics (Pinsky et al. 2008), place the emphasis on high resolution, especially in the vertical, with a measurement accuracy of within an order of magnitude probably sufficient for these purposes. Long time series are necessary for evaluating and improving turbulence schemes in numerical weather prediction models, and the preference here is for robust statistics with low bias.

An active remote sensing approach is required to achieve routine, continuous coverage with simultaneous measurement at all altitudes across a significant part of 
the lower atmosphere, including the full depth of the boundary layer. Doppler radars and lidars can provide the necessary high-resolution velocities and there are a number of methods currently available for estimating the dissipation rate. These broadly fall into three categories: Doppler spectral width, temporal spectra or structure function methods, and conical scanning. An example of a method from each of these categories, as applied to Doppler lidar, is given by Banakh et al. (1999). The methods in these categories may be applicable to both Doppler radar (Brewster and Zrnić 1986; Cohn 1995; Doviak and Zrnić 1993; Chapman and Browning 2001) and Doppler lidar (Gal-Chen et al. 1992; Banakh and Smalikho 1997; Davies et al. 2004). Other possibilities include dual-Doppler lidar (Davies et al. 2005) and radars sensitive to clear-air echoes, which can use the returns arising from turbulent mixing across atmospheric refractive index gradients to estimate dissipation rates (Cohn 1995).

Evaluation of the various methods for estimating dissipation rate from a Doppler lidar or radar is usually performed by comparison with ground-based or towerbased sonic anemometers and sodars (Drobinski et al. 2004). Comparisons with other instruments have also been carried out, such as a lightweight three-dimensional magnetometer carried on a radiosonde (Harrison et al. 2009).

The method of estimating the dissipation rate $\epsilon$ from the Doppler spectral width assumes that turbulence is entirely responsible for the spectral broadening; in practice there are additional sources of spectral broadening that must be accounted for (Doviak and Zrnić 1993), such as wind shear. Not all Doppler lidars provide the full Doppler spectrum, so in this paper we utilize a method that requires only the mean Doppler velocity. We make the assumption that the variations of the mean Doppler velocity over a short sampling time are entirely due to turbulence. Using the variance of a number of samples of the mean Doppler velocity sidesteps most of the issues involved in correcting the various sources of additional spectral broadening associated with the Doppler spectral width method, but, since a longer integration time is required, care must be taken that the scales of turbulent motion now encompassed still remain within the inertial subrange (Frehlich and Cornman 2002).

In this paper we outline a simple method for estimating dissipation rate from unattended continuously operating Doppler lidars. In section 2 we present the method for estimating dissipation rate from the variance of the mean Doppler velocity, with corrections for the expected uncertainty in the observed Doppler velocities for a heterodyne system. An error analysis is given in

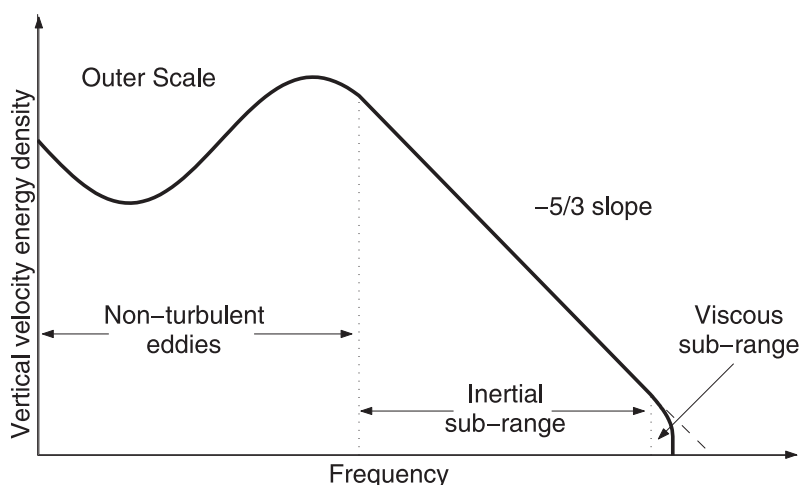

FIG. 1. Schematic of vertical velocity energy density spectra vs frequency conforming to Kolmogorov's hypothesis.

section 3 and validation of the method by comparison with balloon-borne in situ data is presented in section 4 .

\section{Estimating dissipation rate}

\section{a. Standard method from velocity power spectra}

Standard methods for estimating dissipation rate from high-frequency measurements of turbulent velocities typically involve the transformation of the velocity spectra into the frequency domain [e.g., by the use of fast Fourier transforms (FFTs)]. In theory, with sufficient resolution, these vertical velocity spectra are presumed to have a form similar to that shown in Fig. 1, when plotted versus frequency. Production of turbulent kinetic energy (TKE) is dominated by large eddies (length scales of $100 \mathrm{~m}$ or more), which then decay into smaller and smaller eddies (the inertial subrange) until the length scales are small enough for the kinetic energy to be dissipated into heat by molecular diffusion in the viscous subrange (scales on the order of centimeters or less).

In the case of homogeneous and isotropic turbulence, the Kolmogorov (1941) hypothesis states that within the inertial subrange the statistical representation of the turbulent energy spectrum $S(k)$ is given by

$$
S(k)=a \epsilon^{2 / 3} k^{-5 / 3},
$$

where $a=0.55$ is the Kolmogorov constant for onedimensional wind spectra (Paquin and Pond 1971); $\epsilon$ is the dissipation rate; and $k$ is the wavenumber, which can be related to a length scale $L(k=2 \pi / L)$ by invoking Taylor's hypothesis of frozen turbulence (Taylor 1935). If observed spectra fit the form shown in Fig. 1, a $-5 / 3$ power law can be fitted to the portion of the spectrum that lies within the inertial subrange and thus $\epsilon$ can be estimated (e.g., Lothon et al. 2009). 


\section{b. Variance of mean Doppler velocity}

We now introduce a new parameter $\sigma_{\bar{v}}^{2}$, which is the variance of the observed mean Doppler velocity over a defined number of sequential samples $N$ (O'Connor et al. 2005). Initially, we consider the case where the observed variance is dominated by the turbulent processes in the vertical and there are no significant contributions from other sources. The velocity variance is then equivalent (Bouniol et al. 2003) to integrating (1) so that

$$
\begin{aligned}
\sigma_{\bar{v}}^{2} & =\int_{k}^{k_{1}} S(k) d k, \\
& =-\frac{3}{2} a \epsilon^{2 / 3}\left(k_{1}^{-2 / 3}-k^{-2 / 3}\right), \\
& =\frac{3 a}{2}\left(\frac{\epsilon}{2 \pi}\right)^{2 / 3}\left(L^{2 / 3}-L_{1}^{2 / 3}\right),
\end{aligned}
$$

where the wavenumber $k_{1}=2 \pi / L_{1}$ corresponds to the length scale describing the scattering volume dimension for the dwell time of the lidar for a single sample, and $k=2 \pi / L$ relates to the length scale of the large eddies traveling through the lidar beam during the $N$ sampling intervals.

The length scale for an individual sample is given by

$$
L_{1}=U t+2 z \sin \left(\frac{\theta}{2}\right)
$$

where $\theta$ is the half-angle divergence of the lidar beam, $U$ is the horizontal wind, $t$ is the dwell time, and $z$ is the height in meters. Usually, the second term in (5) is negligible as Doppler lidars typically have a very small divergence, $<0.1 \mathrm{mrad}$. Over $N$ sampling intervals, the length scale is $L=N U t$. If the lidar instrument is set to acquire one profile of velocity measurements every $4 \mathrm{~s}$, the length scales for a typical wind speed of $U=10 \mathrm{~m} \mathrm{~s}^{-1}$ in the boundary layer are $L_{1}=40 \mathrm{~m}$ and, if 10 samples are used to calculate $\sigma_{\bar{v}}^{2}, L=400 \mathrm{~m}$. The length scales for horizontal wind speeds as low as $0.25 \mathrm{~m} \mathrm{~s}^{-1}$, with an integration time of $4 \mathrm{~s}$ or greater, should still be much larger than the expected cutoff in the viscous subrange. Assuming both length scales lie within the inertial subrange, we can now write

$$
\epsilon=2 \pi\left(\frac{2}{3 a}\right)^{3 / 2} \sigma_{\bar{v}}^{3}\left(L^{2 / 3}-L_{1}^{2 / 3}\right)^{-3 / 2}
$$

and hence estimate $\epsilon$ directly from $\sigma_{\bar{v}}^{2}$ without the need to calculate FFTs. It should be noted, however, that it is not as easy to determine if the length scales are appropriate in the absence of spectra.

\section{c. Noise contribution to variance}

So far we have assumed that turbulence is the only source of variance. We now consider the influence of noise on the Doppler velocity measurement. The error in an individual Doppler lidar velocity estimate is dependent on the signal-to-noise ratio (SNR) of the measurement. For a heterodyne Doppler lidar, Pearson et al. (2009) have shown that when many pulses have been averaged, the theoretical standard deviation of the Doppler velocity estimate $\sigma_{e}$ for weak signals can be reliably approximated by (Rye and Hardesty 1993)

$$
\begin{aligned}
\sigma_{e}^{2} & =\frac{\Delta v^{2} \sqrt{8}}{\alpha N_{p}}\left(1+\frac{\alpha}{\sqrt{2 \pi}}\right)^{2}, \\
\alpha & =\frac{\operatorname{SNR}}{\sqrt{2 \pi}} \frac{B}{\Delta v},
\end{aligned}
$$

where $\alpha$ is the ratio of the lidar detector photon count to the speckle count (Rye 1979), $\Delta v$ is the signal spectral width, $B$ is the receiver bandwidth (both expressed here in meters per second so that $B$ corresponds to twice the Nyquist velocity), and $N_{p}$ is the accumulated photon count. Both $N_{p}$ and $\alpha$ are determined from the instrument characteristics and the wideband SNR of the target return for a single point sample:

$$
N_{p}=\mathrm{SNR} n M,
$$

where $n$ is the number of pulses averaged per profile and $M$ is the number of points sampled within a specified range gate to obtain a raw velocity. The term wideband SNR refers to the ratio of the average total signal power to the average noise power over the full bandwidth. Note that, because of oversampling and subsequent averaging, the final range gate length does not necessarily coincide with the pulse length.

For a direct detection system, the theoretical minimum for the standard deviation of the Doppler velocity estimate is given by $\sigma_{e}=\Delta v /\left(N_{p}^{0.5}\right)$, although in practice there are additional factors to consider (McKay 1998).

Data from two coherent heterodyne Doppler lidars are presented here. The instruments are very similar in design but have had certain parameters optimized for different objectives. The instrument at Chilbolton has a longer integration time to improve sensitivity as it is configured for a primary function of observing liquid and ice cloud at all heights up to $10 \mathrm{~km}$. The instrument deployed for the second Regent's Park and Tower Environmental Experiment (REPARTEE) campaign in central London (Martin et al. 2009) is optimized for boundary layer studies and has achieved the required sensitivity with a shorter integration time by having the 
TABLE 1. Doppler lidar specifications. The instrument deployed during the 2nd REPARTEE experiment in London is essentially the same as the one deployed at Chilbolton, but with certain parameters adjusted to maximize the measurement capabilities within the boundary layer. Where particular parameters differ, the REPARTEE instrument parameters are given in parentheses. Both instruments were built by Halo Photonics.

\begin{tabular}{ll}
\hline \hline \multicolumn{1}{c}{ Wavelength } & \multicolumn{1}{c}{$1.5 \mu \mathrm{m}$} \\
\hline Pulse repetition rate & $\left.15 \mathrm{kHz}(20 \mathrm{kHz})_{\text {Nyquist velocity }} \mathrm{m} \mathrm{s}^{-1}(14 \mathrm{~m} \mathrm{~s})^{-1}\right)$ \\
Sampling frequency & $50 \mathrm{MHz}^{\circ}(30 \mathrm{MHz})$ \\
Points per range gate & $12(6)$ \\
Pulses averaged & 20000 \\
Raw profiles averaged & $5(1)$ \\
Range resolution & $36 \mathrm{~m}(30 \mathrm{~m})$ \\
Integration time & $30 \mathrm{~s}(4 \mathrm{~s})$ \\
Pulse duration & $0.2 \mu \mathrm{s}$ \\
Lens diameter & $6 \mathrm{~cm}(8 \mathrm{~cm})$ \\
Divergence & $33 \mu \mathrm{rad}$ \\
Focus & $\infty(801 \mathrm{~m})$ \\
Telescope & Monostatic optic-fiber coupled \\
\hline
\end{tabular}

telescope focus set to approximately $800 \mathrm{~m}$ (note that this reduces the instrument sensitivity dramatically above $2 \mathrm{~km}$ ). The specifications of the two Doppler lidar instruments are given in Table 1 . The pulse length for both instruments is the same, $30 \mathrm{~m}$, but the signals are oversampled (by a factor of 10 for the instrument at Chilbolton and by 6 for the REPARTEE instrument). These highresolution samples, or points, are then averaged up to yield the raw velocity estimates at the selected range gate length; the number averaged is given by the number of points per range gate parameter in Table 1 . The acquisition time for a single profile obtained from 20000 pulses is dependent on the pulse repetition rate of the instrument and additional time is then required for realtime computation of the velocities; Chilbolton requires approximately $1.33 \mathrm{~s}$ for acquisition and $4 \mathrm{~s}$ for computation per profile, REPARTEE requires $1 \mathrm{~s}$ for acquisition plus $3 \mathrm{~s}$ for computation per profile. The Chilbolton instrument then performs additional averaging of five profiles to give a total integration time of about $30 \mathrm{~s}$.

We first consider the REPARTEE instrument as it is potentially more suitable for estimating dissipation rate because of its much shorter integration time. The theoretical standard deviation of the Doppler velocity estimate as a function of wideband SNR is given in Fig. 2 for the REPARTEE instrument. It is immediately obvious that the relationship between $\sigma_{e}$ and wideband SNR for a heterodyne system is not the same as that for a direct detection system. In fact, at $-25 \mathrm{~dB}, \sigma_{e}$ is an order of magnitude higher. It is also apparent that, for a heterodyne system, once the wideband SNR has reached $0 \mathrm{~dB}$, increasing the SNR further does not greatly improve $\sigma_{e}$ [see Rye and Hardesty (1993) for a comprehensive explanation]. The choice of signal spectral width, $\Delta v$, also has some influence on estimating $\sigma_{e}$. Pearson et al. (2009) suggested a value of $1.5 \mathrm{~m} \mathrm{~s}^{-1}$ for $\Delta v$, our results indicate that $2 \mathrm{~m} \mathrm{~s}^{-1}$ is more suitable and we select this as a typical value for the rest of the paper.

We first investigate observed vertical-velocity energy density spectra to confirm that they have the same shape as the idealized form given in Fig. 1, to examine the noise contribution, and to note whether the spectra contain a sufficient portion within the inertial subrange for (6) to be valid. Data from the REPARTEE instrument has been selected because the shorter integration time allows the spectra to encompass smaller scales. Figures 3-5 display vertical-velocity energy density spectra at three different heights $(135,825$, and $1275 \mathrm{~m})$ calculated from 60 min of data over three adjacent gates (approximately 2700 individual velocity estimates). The targets in all cases are aerosol particles in the boundary layer. If velocity measurements and their random estimation error are uncorrelated, then Frehlich (2001) states that the temporally uncorrelated estimation error will appear as a constant-amplitude high-frequency region in the velocity spectra. The noise contribution is computed as the ensemble mean $\left\langle\sigma_{e}\right\rangle$ of the individual estimates of $\sigma_{e}$ calculated for each individual measurement using (7). In terms of vertical-velocity energy density spectra, the levels of the theoretical noise contribution $\left\langle\sigma_{e}\right\rangle^{2}$ are then scaled by dividing by the frequency span of the spectrum (approximately $0.125 \mathrm{~Hz}$ ) to obtain the noise variance displayed as dashed lines in Figs. 3-5.

The vertical-velocity energy density spectrum in Fig. 3 was obtained from data with a mean SNR close to $-7 \mathrm{~dB}$ and there is no indication of noise at the high end of the frequency spectrum, which is consistent with a very low theoretical noise level of $0.009 \mathrm{~m}^{2} \mathrm{~s}^{-1}$. This spectrum has the same form as the idealized version given in Fig. 1 and a $-5 / 3$ power law can be fitted to the high frequency end of the spectrum (from approximately $1 \times$ $10^{-2} \mathrm{~Hz}$ to the Nyquist frequency; $0.125 \mathrm{~Hz}$ ). The horizontal wind speeds given by the Doppler lidar in scanning mode, and the Met Office North Atlantic and European (NAE) operational numerical weather prediction model, were both close to $2 \mathrm{~m} \mathrm{~s}^{-1}$ at $135 \mathrm{~m}$ and 0800 UTC. The frequency range therefore corresponds to a spatial range of approximately $200-16 \mathrm{~m}$ and implies that the transition from the inertial subrange to the outer scale for nonturbulent eddies occurs at length scales of $200 \mathrm{~m}$. From a $-5 / 3$ power-law fit to this frequency range we calculate $\epsilon=3 \times 10^{-3} \mathrm{~m}^{2} \mathrm{~s}^{-3}$.

The energy density spectrum in Fig. 4 was obtained from data with a mean SNR of $-14 \mathrm{~dB}$, for which the theoretical noise level is $0.12 \mathrm{~m}^{2} \mathrm{~s}^{-1}$. This agrees very 


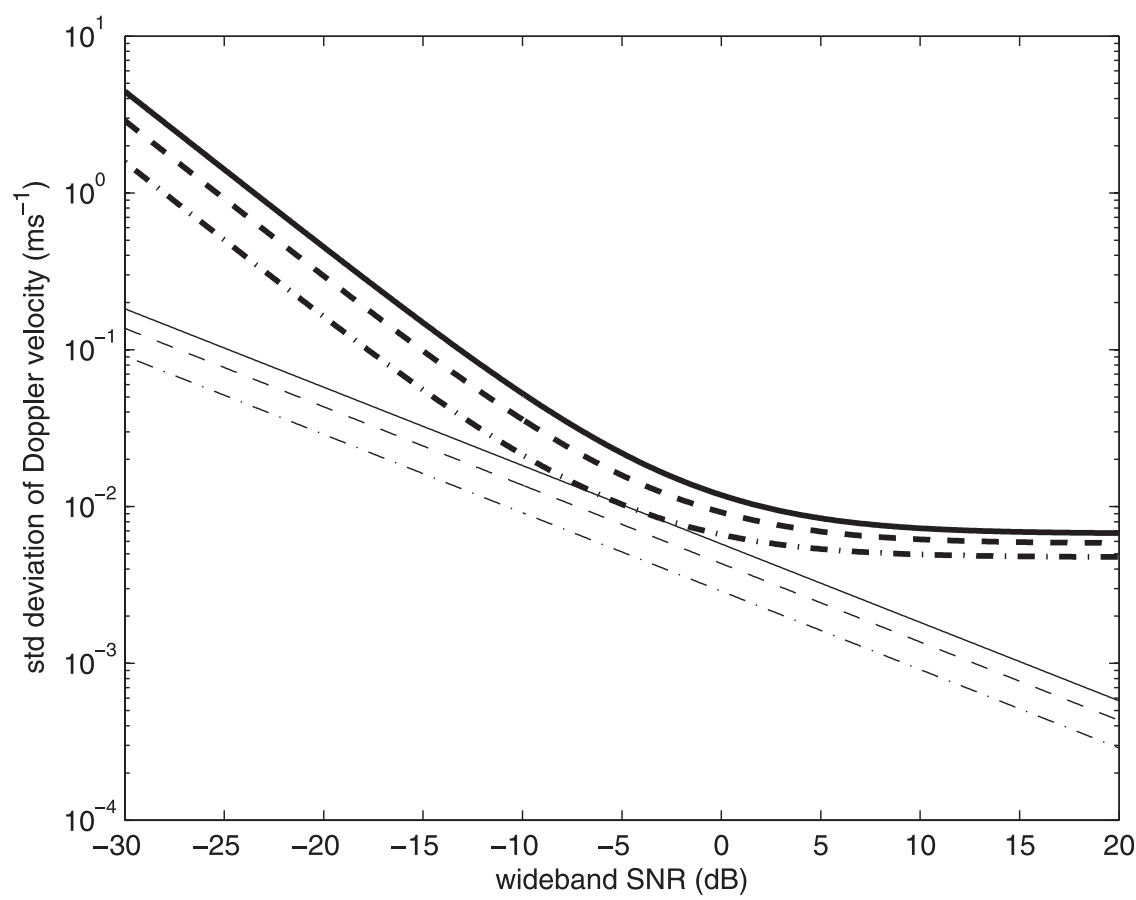

FIG. 2. Theoretical standard deviation of Doppler velocity estimate for the REPARTEE heterodyne Doppler lidar (thick lines) for three signal spectral widths, equivalent to 1 (dotdashed), 1.5 (dashed), and 2 (solid) $\mathrm{m} \mathrm{s}^{-1}$, computed using Eqs. (7) and (8). Also shown is the theoretical standard deviation of Doppler velocity estimates for direct detection lidar systems (thin lines). The wideband SNR refers to the ratio of the average total signal power to the average noise power over the full bandwidth for the target return from a single pulse.

well with the observed spectrum, which abruptly flattens out above $10^{-2} \mathrm{~Hz}$, and is consistent with the explanation given by Frehlich (2001). A -5/3 power law can still be fitted to the portion of the spectrum from $4 \times 10^{-3}$ to $1 \times 10^{-2} \mathrm{~Hz}$, which, since the horizontal wind speed at $825 \mathrm{~m}$ is about $6 \mathrm{~m} \mathrm{~s}^{-1}$, corresponds to a spatial range of approximately $1500-600 \mathrm{~m}$. The transition from the inertial subrange to outer scale at this height is at length scales of about $1500 \mathrm{~m}$ and, from the $-5 / 3$ power-law fit, $\epsilon=2 \times 10^{-6} \mathrm{~m}^{2} \mathrm{~s}^{-3}$.

In Fig. 5, the energy density spectrum was derived from data with a mean SNR of about $-20 \mathrm{~dB}$. The noise dominates the spectrum in this case, and is consistent with a theoretical noise level of $5 \mathrm{~m}^{2} \mathrm{~s}^{-1}$. Again, the observed spectrum has a constant amplitude characteristic of temporally uncorrelated estimation noise. It is not reasonable to attempt to fit a $-5 / 3$ power law to any portion of this particular spectrum.

If it is assumed that the sources of variance have a Gaussian distribution and are independent of one another, the observed variance, $\sigma_{\bar{v}}^{2}$, is the sum of the variances from each source (Doviak and Zrnić 1993; Frehlich et al. 1998) such that

$$
\sigma_{\bar{v}}^{2}=\sigma_{\bar{w}}^{2}+\sigma_{e}^{2}+\sigma_{d}^{2},
$$

where $\sigma_{\bar{w}}^{2}$ is the contribution from air turbulence that we are interested in, the contribution from noise is $\sigma_{e}^{2} \approx$ $\left\langle\sigma_{e}\right\rangle^{2}$, and $\sigma_{d}^{2}$ is the contribution from the variation in still-air terminal fall speeds of particulates within the measurement volume from one sample to the next. Because aerosol particles and liquid cloud droplets have terminal fall speeds $<1 \mathrm{~cm} \mathrm{~s}^{-1}$, the variance $\sigma_{d}^{2}$ can be safely ignored for returns from these targets. Figures 3-5 show that the variance arising from the uncertainty in the Doppler velocity measurements can be estimated reliably and that it is valid to assume that the two sources of variance, turbulence and estimator noise, are independent. Thus, given an observed total variance and a calculated noise variance, $\epsilon$ can be derived using (6) by replacing the theoretical $\sigma_{\bar{v}}^{2}$ with $\sigma_{\bar{w}}^{2}=\sigma_{\bar{v}}^{2}-\sigma_{e}^{2}$. Targets such as rain or ice particles will have significant terminal fall speeds; therefore, it may be necessary to quantify $\sigma_{d}^{2}$ when attempting to calculate $\epsilon$ in such situations.

\section{Error in derived dissipation rates}

To estimate the error in $\epsilon$ we first assume that $L_{1} \ll L$ in (6), so that $\epsilon \propto \sigma_{\bar{w}}^{3} / L$, and through the propagation of errors, the fractional error in $\epsilon$ is 


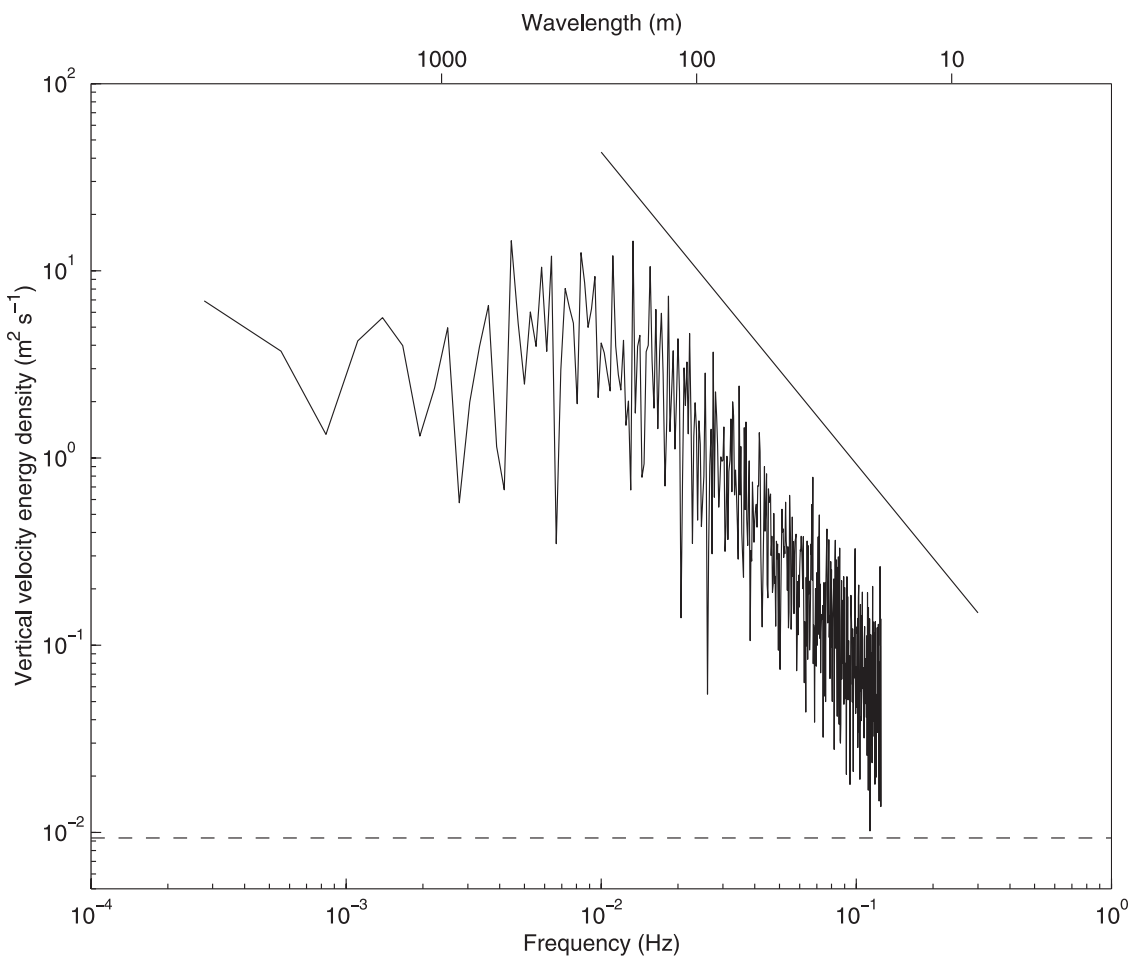

FIG. 3. Power spectra of $60 \mathrm{~min}$ of data with a time resolution $4 \mathrm{~s}$, centered on 0800 UTC 29 Oct 2007, at a height of $135 \mathrm{~m}$ (average taken from three adjacent range gates at 105-165 m). Also shown is the expected noise contribution (dashed line) calculated from the mean SNR of $-7 \mathrm{~dB}$, and a reference $-5 / 3$ power law (solid line). Data are from the REPARTEE instrument.

$$
\frac{\Delta \epsilon}{\epsilon}=\frac{3 \Delta \sigma_{\bar{w}}}{\sigma_{\bar{w}}}+\frac{\Delta L}{L} .
$$

Radiosonde, tower measurements, or wind profilers can be used to estimate the horizontal length scale, as can the output from an operational forecast model. In this study, we use model winds from an operational forecast model to derive $L$ and, since the horizontal winds from the Met Office mesoscale model are generally accurate to $1-2 \mathrm{~m} \mathrm{~s}^{-1}$ (Panagi et al. 2001), we estimate the fractional error in $L$ to be about $10 \%$ for a typical horizontal wind speed of $10 \mathrm{~m} \mathrm{~s}^{-1}$. The extremely small divergence of the Doppler lidar in this study $(0.033 \mathrm{mrad})$ means that the second term in (5) can be ignored even at very low $U$ and short observation times.

Following Lenschow et al. (2000) we can estimate the measurement error in a variance as follows:

$$
\Delta \sigma_{\bar{w}}^{2} \approx \sigma_{\bar{w}}^{2} \sqrt{\frac{4}{N} \frac{\sigma_{e}^{2}}{\sigma_{\bar{w}}^{2}}},
$$

and therefore provide the fractional error for each individual estimate of $\epsilon$. It should be noted that (12) assumes that each velocity sample used to calculate the variance has a similar error to the ensemble mean error, $\sigma_{e} \approx$ $\left\langle\sigma_{e}\right\rangle$. This assumption can be tested and those variances for which this is no longer approximately true should be flagged as unreliable.

Estimates of $\epsilon$ derived from the REPARTEE Doppler lidar data are shown in Fig. 6, along with the derived $\left\langle\sigma_{e}\right\rangle$ and fractional error in dissipation rate, for the same day as in Figs. 3-5. The REPARTEE instrument has a maximum range of about $2 \mathrm{~km}$ and, as Fig. $6 \mathrm{a}$ indicates, is sufficiently sensitive to detect aerosol (or clouds) at almost all ranges, potentially providing an estimate of dissipation rate throughout most of the lower atmosphere.

The convective boundary layer is clearly visible in Fig. 6c, by noting where $\epsilon$ is high, in this case $>10^{-4} \mathrm{~m}^{2} \mathrm{~s}^{-3}$. From midnight (local time is UTC) to 0730 UTC, the well-mixed layer reaches from the surface to about $250 \mathrm{~m}$; it then begins to grow from 0730 UTC and the convective boundary layer top reaches $1.5 \mathrm{~km}$ at 1400 UTC. Maximum values of $\epsilon$ approach $5 \times 10^{-3} \mathrm{~m}^{2} \mathrm{~s}^{-3}$. The convective boundary layer then decays after 1600 UTC and returns to a shallow well-mixed layer that again reaches from the surface to about $250 \mathrm{~m}$. 


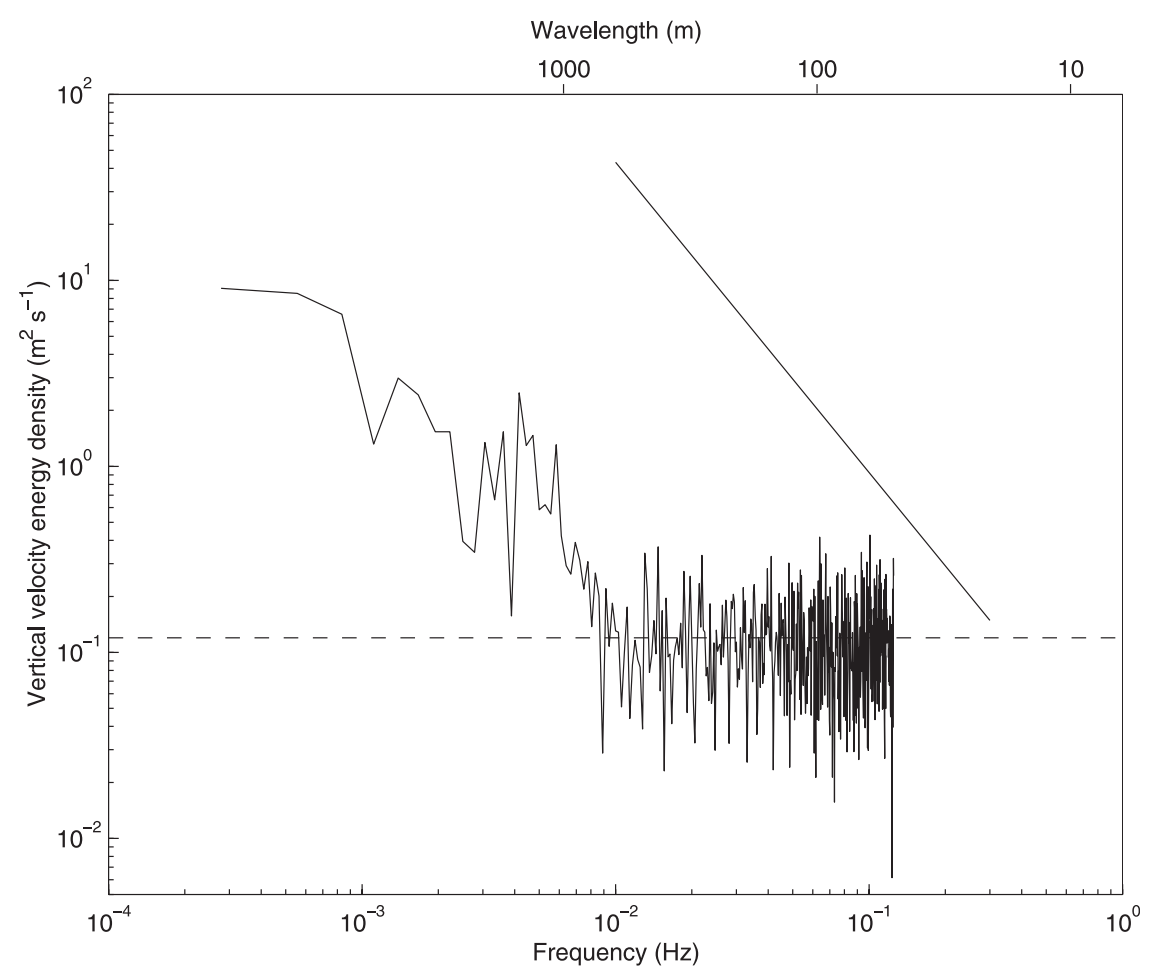

FIG. 4. As in Fig. 3, but at a height of $825 \mathrm{~m}$. The mean SNR is $-14 \mathrm{~dB}$.

Within the convective boundary layer, the limiting factor in providing accurate estimates of $\epsilon$ is the uncertainty in the horizontal winds used to estimate the length scales. The wind speed was $2 \mathrm{~m} \mathrm{~s}^{-1}$ in Fig. 3 , and $6 \mathrm{~m} \mathrm{~s}^{-1}$ in Fig. 4; in the absence of lidar-derived winds, the resulting relative errors in $L$ should be increased from $10 \%$ to $75 \%$ and $25 \%$, respectively. Values of the ensemble mean of the theoretical error in the observed velocities are $<0.1 \mathrm{~m} \mathrm{~s}^{-1}$ throughout much of the lowest $1 \mathrm{~km}$ (Fig. 6b). However, the resulting error variance, $\sigma_{e}^{2}$, although small, may still be a substantial fraction of the observed $\sigma_{\bar{v}}^{2}$. As suggested by the expression (12), this can be mitigated by increasing the number of samples used in calculating $\epsilon$ until the fractional error, $\Delta \sigma_{\bar{w}} / \sigma_{\bar{w}}$, is reduced to an acceptable level. The number of samples used in calculating Fig. 6 was 45 (equivalent to $3 \mathrm{~min}$ ), which resulted in a fractional error in $\sigma_{\bar{w}}$ of about $10 \%$ within the convective boundary layer.

Outside the convective boundary layer, uncertainty in the velocity variance estimates is much more likely to be the dominant source of error; a smaller velocity variance due to less turbulent conditions is compounded by more uncertainty in the variance estimate due to low SNR. Figures $6 b, d$ corroborate this. Within the convective boundary layer, where $\epsilon$ is high, the fractional error in $\epsilon$ is estimated to be as low as $30 \%$. For quiescent conditions in early morning or late evening with similar mean errors in velocity, the derived fractional error in $\epsilon$ is at least $100 \%$. Fractional errors in $\sigma_{\bar{w}}>100 \%$ indicate where noise is the dominant source of variance; from (11) this translates to fractional errors in $\epsilon$ of over $300 \%$ and provides a quality flag for identifying unreliable $\epsilon$ estimates. Such values are found, for example, for locations within the time-height period (0730-0830 UTC and $1230-1320 \mathrm{~m}$ ) used for generating Fig. 5, whose noisy spectrum does not show any sign of an inertial subrange.

Thus, by limiting retrievals to estimates with fractional errors in $\epsilon<300 \%$, reliable estimates of $\epsilon$ can be derived to within at least an order of magnitude throughout almost all of the lowest $2 \mathrm{~km}$ and, in the convective boundary layer, to within $50 \%$ or better for this instrument.

In providing these error estimates, we have also implicitly assumed that the sampling time is sufficiently short to ensure that we remain in the inertial subrange, where (1) applies. If the sampling time is too long, then the observed velocity variance will include contributions from the outer scales of turbulence, where (1) no longer applies, and $\epsilon$ will be underestimated. For this case, the horizontal winds used to derive $\epsilon$ were taken from the Met Office NAE model and ranged from 2 to $6 \mathrm{~m} \mathrm{~s}^{-1}$. With $N=45$ consecutive 4-s samples used to calculate $\sigma_{\bar{v}}^{2}$, this corresponds to length scales for $L$ of $360-1080 \mathrm{~m}$, which, according to Figs. 3 and 4, suggests that the 


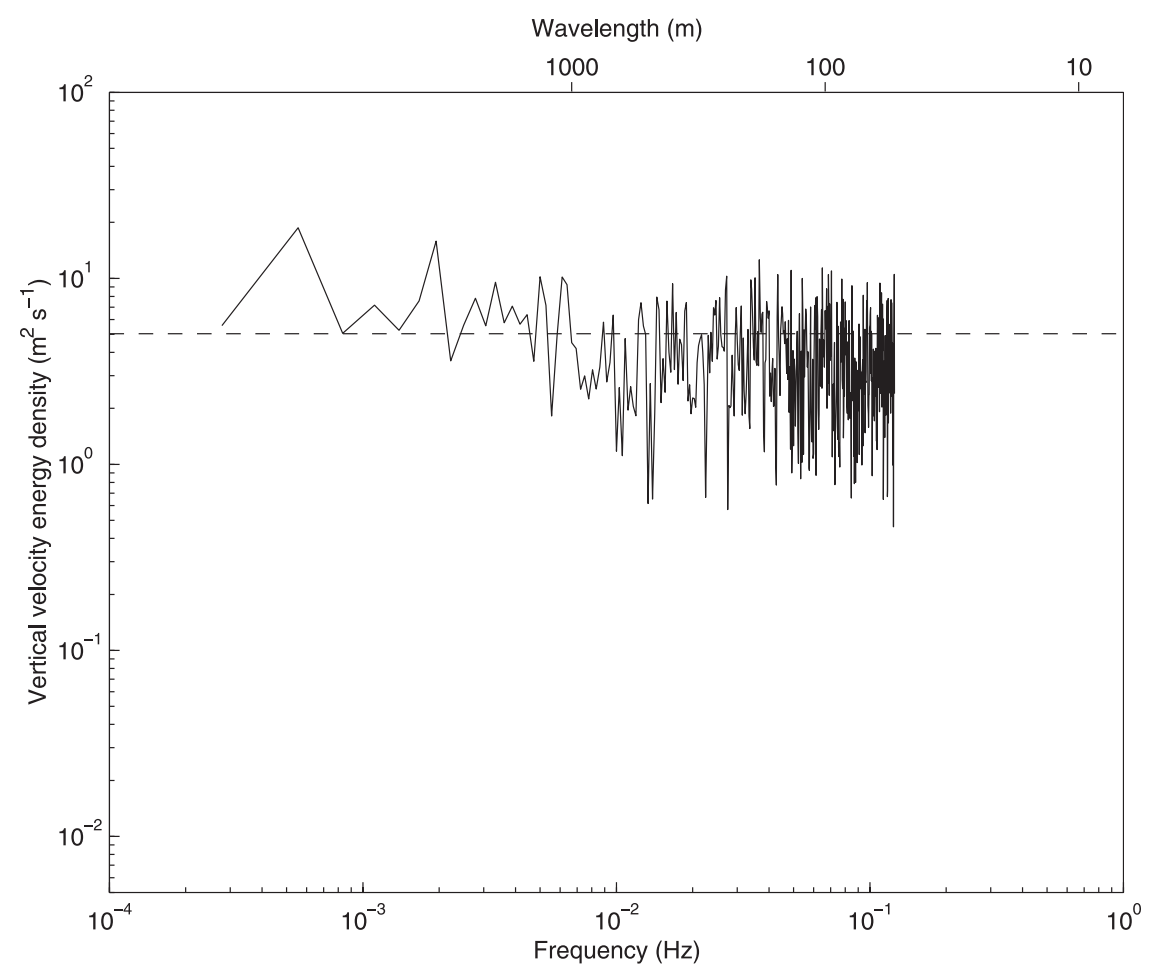

FIG. 5. As in Fig. 3, but at a height of $1275 \mathrm{~m}$. The mean SNR is $-20 \mathrm{~dB}$.

assumption is reasonable at ranges near to the surface (although possibly not when very close to the surface) and is valid at greater ranges. The length scales for $L_{1}$, 36-108 $\mathrm{m}$, are substantially greater than the transition from the inertial subrange to the viscous subrange. At high levels of turbulence within the well-mixed boundary layer the sampling time of this Doppler lidar is sufficiently fast to acquire enough samples while remaining in the inertial subrange, but this may no longer be true in very quiescent conditions above the well-mixed boundary layer. In these cases the value of $\epsilon$ can be severely underestimated.

\section{Balloon-borne in situ evaluation}

We now present estimates of the dissipation rate for data taken from the Doppler lidar at Chilbolton on 22 April 2008. Appropriate parameters for this day are given in Fig. 7. Low cloud or fog at $300 \mathrm{~m}$ height is present from 0400 to 0800 UTC and completely attenuates the lidar signal (Fig. 7a), but otherwise there is potential coverage throughout most of the lowest $2 \mathrm{~km}$. A convective boundary layer is again evident in Fig. 7c, from about 1000 to 1600 UTC, which grows to over $1.5 \mathrm{~km}$ with $\epsilon$ values reaching $5 \times 10^{-2} \mathrm{~m}^{2} \mathrm{~s}^{-3}$. Similar to the REPARTEE case, the horizontal winds taken from the Met Office NAE model again ranged from 2 to
$6 \mathrm{~m} \mathrm{~s}^{-1}$, but, since the Chilbolton instrument has a longer integration time to improve the sensitivity, while $N=10$ consecutive 30 -s samples were used to calculate $\sigma_{\bar{v}}^{2}$, the length scales for $L$ are larger, at $600-1800 \mathrm{~m}$. The advantage of this longer integration time is clearly visible in Fig. 7b, where the increase in accumulated photocount $N_{p}$ leads to lower theoretical errors in velocity for a similar SNR.

Since these length scales may now incorporate unwanted contributions from the outer scale as well as the inertial subrange, especially when close to the surface, we also performed calculations using $N=12$ samples taken from four consecutive rays in time and from three adjacent gates in height. The length scales for L using this approach are potentially more applicable, at 240$720 \mathrm{~m}$, although again probably too large close to the surface. The absolute values of $\epsilon$ are not exactly the same as those in Fig. 7c, but the pertinent features of such a figure are very similar and so not included here. Within the convective boundary layer, the histogram of $\epsilon$ has approximately the same shape and mean, indicating that the length scales for both sampling regimes do remain within the inertial subrange. Examining Fig. 1 reminds us that we would expect $\epsilon$ estimates in the sampling regime with length scales remaining within the inertial subrange to be larger than those with length scales extending into the outer range. This explains why values 

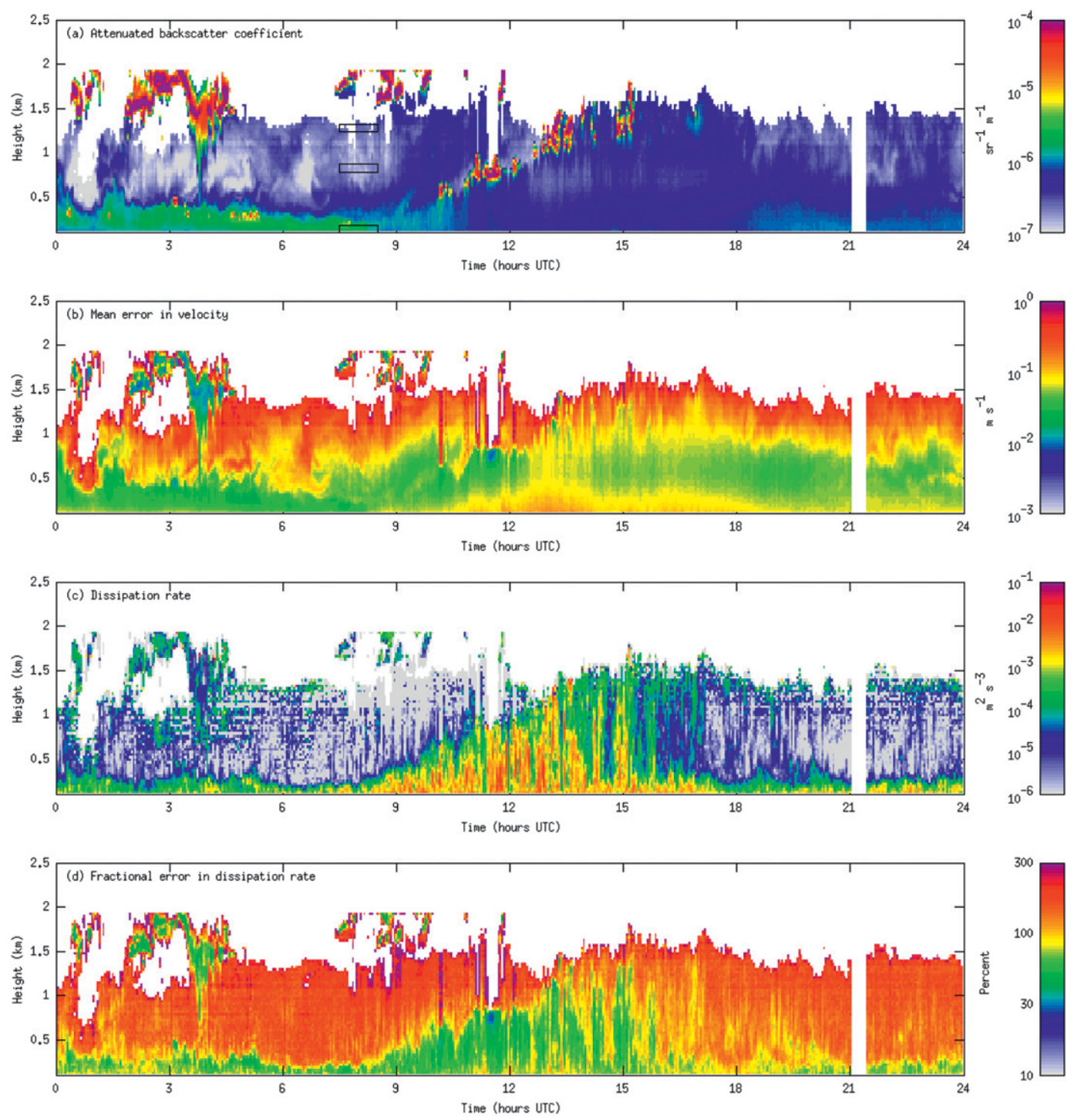

FIG. 6. (a) Attenuated backscatter coefficient from the Doppler lidar during the REPARTEE campaign in London on 29 Oct 2007. The three boxes centered on 0800 UTC indicate the locations for the data presented in Figs. 3-5. (b) The ensemble mean of the theoretical uncertainty in observed velocities calculated using Eq. (6) for each sample used to derive (c) the dissipation rate. (d) The estimated fractional error in dissipation rate, calculated using Eq. (11).

of $\epsilon$ outside of the convective boundary layer are often larger than those in Fig. 7c, sometimes by as much as factor of 5 .

During April 2008 the University of Leeds Universities' Facility for Atmospheric Measurement (UFAM) SkyDoc balloon was flown at Chilbolton in close proximity to the Doppler lidar carrying a turbulence sonde and mean meteorology instrumentation. The turbulence sonde is a prototype instrument incorporating the sensing head and control electronics of a Gill Windmaster three-axis sonic anemometer in a compact aerofoil housing. The sonic anemometer measures the three components of the turbulent wind at $40 \mathrm{~Hz}$ and internally averages the measurements to $10 \mathrm{~Hz}$ before outputting the data via a serial interface. The data stream was logged via a compact embedded Linux computer installed in the housing along with a $12-\mathrm{V}$ battery pack. A separate enclosure housed a mean meteorology package to measure air pressure, mean temperature and relative humidity, and mean wind speed; a compact aerosol probe (CLASP; Hill et al. 2008) was also included to measure aerosol size spectra for a related study. These instruments were suspended approximately $20 \mathrm{~m}$ below the balloon, which is about $3.5 \mathrm{~m}$ in diameter when inflated.

For calculation of the dissipation rate we select the portion of the power spectrum at frequencies greater 

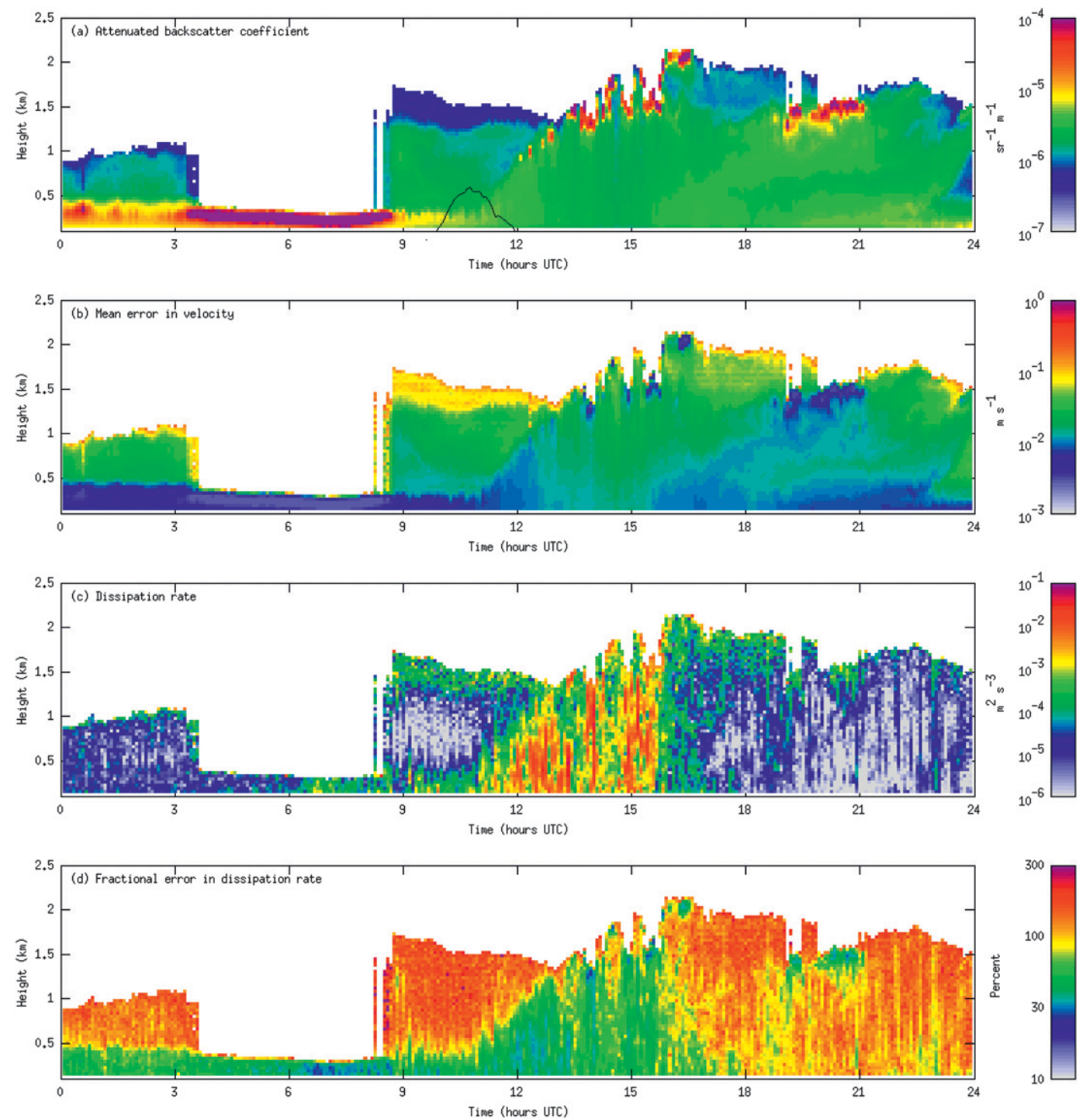

FIG. 7. (a) Attenuated backscatter coefficient from the Doppler lidar at Chilbolton for 22 Apr 2008. (b) The ensemble mean of the theoretical uncertainty in observed velocities calculated using Eq. (11) for each sample used to derive (c) the dissipation rate. (d) The estimated fractional error in dissipation rate, calculated using Eq. (11). The black line (from about 1000 to 1200 UTC) denotes the balloon flight track.

than $2 \mathrm{~Hz}$. This limit is chosen to avoid that part of the spectra contaminated by the motion of the tethersonde; this spans a frequency range of approximately 0.08 $0.2 \mathrm{~Hz}$. It should be noted that, for a $4 \mathrm{~m} \mathrm{~s}^{-1}$ horizontal wind speed, frequencies from 2 to $10 \mathrm{~Hz}$ correspond to length scales from $2 \mathrm{~m}$ down to $40 \mathrm{~cm}$, which, although above the viscous subrange, are significantly smaller than the length scales probed by the lidar.

The flight track of the balloon is superimposed on the plots in Fig. 7 and the weight of the turbulence sonde limited the maximum height to about $600 \mathrm{~m}$. Lidar estimates of $\epsilon$ closest to the location of the balloon in height and time were selected for comparison. The balloon is obviously not collocated exactly with the lidar beam, and depending on wind conditions, may be as much as $400 \mathrm{~m}$ away in the horizontal. We consciously used model forecast horizontal winds to estimate the Doppler lidar $\epsilon$ values (whereas, the balloon values were calculated from in situ measurements of the horizontal wind) so that we could examine how the lidar technique would perform in an operational context.

A comparison of the in situ measurements of $\epsilon$ with those inferred from the Doppler lidar shows good general agreement both in time, Fig. 8, and in height, Fig. 9. The balloon observes a significant decrease in $\epsilon$ with height of over three orders of magnitude; the lidar 


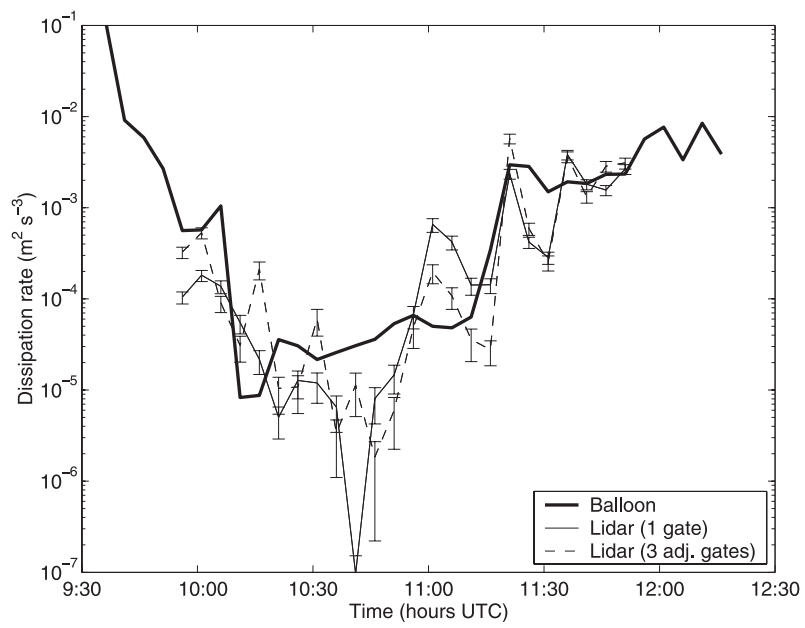

FIG. 8. Observed rate of dissipation of TKE, $\epsilon$, in the boundary layer from the lidar and in situ balloon measurements between 100 and $600 \mathrm{~m}$ on 22 Apr 2008.

method captures this decrease, and is also able to cover the wide variation in magnitude. For values close to the ground, between 100 and $250 \mathrm{~m}$, better agreement is found between the balloon and lidar estimates derived from four consecutive samples and three adjacent gates.

Concerns noted earlier about the length scales involved near the surface being too large when 10 consecutive samples at one gate are used to derive the lidar estimates are most likely responsible for this discrepancy. The values of lidar $\epsilon$ below $10^{-6} \mathrm{~m}^{2} \mathrm{~s}^{-3}$ are those that display the greatest disagreement with the balloon measurements and are again due to the limitations in using 10 consecutive samples at one gate. As shown in Fig. 9, this occurs at a height of almost $600 \mathrm{~m}$, which, at 1040 UTC, is not yet encompassed by the growing convective boundary layer. Two factors limit the ability of the lidar to estimate $\epsilon$ in this particular case; not only is the lidar SNR low above the convective boundary layer, the length scales over which the velocity variance is calculated are again unlikely to be wholly contained within the inertial subrange. Using four consecutive samples and three adjacent gates to estimate $\epsilon$ at this height does show considerable improvement, but it is still not certain that the shorter length scales involved will remain within the inertial subrange outside the convective boundary layer.

One method of diagnosing whether the length scales probed by the lidar only encompass the inertial subrange is to vary the number of samples $N$ used to calculate $\epsilon$. If the derived values of $\epsilon$ are no longer consistent with each other, then the probability is high that the observed variance $\sigma_{\bar{v}}^{2}$ contains contributions from the outer scales of turbulence.

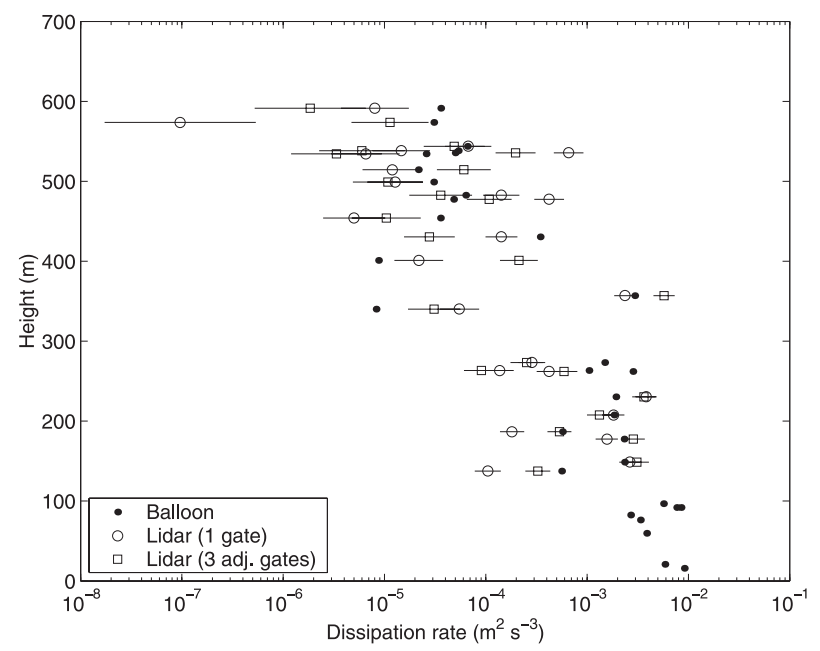

FIG. 9. Observed rate of dissipation of TKE, $\epsilon$, in the boundary layer from the lidar and in situ balloon measurements between 0930 and 1230 UTC 22 Apr 2008.

\section{Conclusions}

We have demonstrated the potential for estimating $\epsilon$ from a Doppler lidar by using the standard deviation of successive samples of the mean Doppler velocity. We have shown that the noise contribution to the velocity variance can be estimated reliably and that there is sufficient SNR throughout most of the well-mixed boundary layer for good Doppler observations. The range of values found for $\epsilon$ agree well with the wide range of dissipation rates measured by Chen (1974) and Siebert et al. (2006). The agreement with the in situ balloonborne measurements is very encouraging; however, it should be noted that this comparison was mainly performed within the well-mixed boundary layer, where the lidar signals are generally strong and the scales of motion contained within the inertial subrange are large enough to encompass the entire $N$ sampling times required to derive $\sigma_{\bar{v}}$.

In principle the retrieval of $\epsilon$ in low SNR conditions can be improved by discarding the individual Doppler velocity measurements with large errors before computing $\sigma_{\bar{v}}$, as discussed by Frehlich (2001). For lowpower Doppler lidars, which require averaging of many pulses to achieve a reasonable sensitivity, there are a limited number of individual samples available within the required timeframe for keeping length scales within the inertial subrange, and it is highly likely that removal of the noisy samples will bias the calculation of $\epsilon$. A threshold on the relative frequency of noisy samples within a variance measurement provides a simple quality flag for the $\epsilon$ estimates.

It is clear that a shorter integration time is preferable for ensuring that the length scales probed are always 
within the inertial subrange to ensure that (1) is applicable. The boundary between the outer scale and the inertial subrange may well lie at much smaller scales in some regions of the atmosphere (and in some types of boundary layers). However, for the particular instruments considered here, there is a trade-off between the sensitivity of the instrument and the applicability of the method. This method can still be applied where longer integration times have been used to improve the instrument sensitivity, but there will be more situations when the length scales are no longer within the inertial subrange. From the measurements discussed here it seems that an instrument temporal resolution of $4 \mathrm{~s}$, resulting in a $\sigma_{\bar{v}}$ estimate over $<120 \mathrm{~s}$, is required to remain within the inertial subrange throughout the boundary layer; whereas, an instrument with a temporal resolution of $30 \mathrm{~s}$ may be limited to the length scales found in convective boundary layers. In contrast, the ability of the instrument to measure very low velocity variances with sufficient accuracy is much improved as the accumulated photon count increases, necessitating the extended integration time. With 30 -s samples there is potential for reducing length scales by taking samples from adjacent gates so that the number of consecutive samples in time can be reduced accordingly, while still providing estimates of dissipation rates with reasonable accuracy.

Acknowledgments. We acknowledge the FGAM facility for providing the $1.5-\mu \mathrm{m}$ Doppler lidar data from the REPARTEE project and the Met Office for providing the Met Office North Atlantic and European (NAE) model data. The 1.5- $\mu \mathrm{m}$ Doppler lidar at Chilbolton was acquired with NERC Grant NE/C513569/1 and operated and maintained by the Rutherford Appleton Laboratory. We also thank Sarah Norris and Cathryn Birch for operating the University of Leeds SKYDOC UFAM balloon, which was flown in April 2008 under NERC Grant NE/F010338/1.

\section{REFERENCES}

Banakh, V. A., and I. N. Smalikho, 1997: Estimation of turbulent energy dissipation rate from data of pulse Doppler lidar. At mos. Oceanic Opt., 10, 957-965.

— — _ _ F. Köpp, and C. Werner, 1999: Measurements of turbulent energy dissipation rate with a CW Doppler lidar in the atmospheric boundary layer. J. Atmos. Oceanic Technol., 16, 1044-1061.

Bouniol, D., A. J. Illingworth, and R. J. Hogan, 2003: Deriving turbulent kinetic energy dissipation rate within clouds using ground based $94 \mathrm{GHz}$ radar. Preprints, 31st Conf. on Radar Meteorology, Seattle, WA, Amer. Meteor. Soc., 193-196.

Brewster, K. A., and D. S. Zrnić, 1986: Comparison of eddy dissipation rates from spatial spectra of Doppler velocities and Doppler spectrum widths. J. Atmos. Oceanic Technol., 3, 440-452.
Caughey, S. J., and S. G. Palmer, 1979: Some aspects of turbulence structure through the depth of the convective boundary layer. Quart. J. Roy. Meteor. Soc., 105, 811-827.

_ J. Wyngaard, and J. Kaimal, 1979: Turbulence in the evolving stable boundary layer. J. Atmos. Sci., 36, 1041-1052.

Chapman, D., and K. Browning, 2001: Measurements of dissipation rate in frontal zones. Quart. J. Roy. Meteor. Soc., 127, 1939-1959.

Chen, W. Y., 1974: Energy dissipation rates of free atmospheric turbulence. J. Atmos. Sci., 31, 2222-2225.

Cohn, S. A., 1995: Radar measurements of turbulent eddy dissipation rate in the troposphere: A comparison of techniques. J. Atmos. Oceanic Technol., 12, 85-95.

Davies, F., C. G. Collier, G. N. Pearson, and K. E. Bozier, 2004: Doppler lidar measurements of turbulent structure function over an urban area. J. Atmos. Oceanic Technol., 21, 753-761.

- - - and K. E. Bozier, 2005: Errors associated with dual Doppler lidar turbulence measurements. J. Opt. A, 7, 280-289.

Doviak, R. J., and D. S. Zrnić, 1993: Doppler Radar and Weather Observations. 2nd ed. Academic Press, 562 pp.

Drobinski, P., R. A. Brown, P. H. Flamant, and J. Pelon, 2004: Evidence of organized large eddies by ground-based Doppler lidar, sonic anemometer and sodar. Bound.-Layer Meteor., 88, 343-361.

Fairall, C. W., R. Markson, G. E. Schacher, and K. L. Davidson, 1980: An aircraft study of turbulence dissipation rate and temperature structure function in the unstable marine atmospheric boundary layer. Bound.-Layer Meteor., 18, 453-469.

Frehlich, R., 2001: Estimation of velocity error for Doppler lidar measurements. J. Atmos. Oceanic Technol., 18, 1628-1639.

, and L. Cornman, 2002: Estimating spatial velocity statistics with coherent Doppler lidar. J. Atmos. Oceanic Technol., 19, 355-366.

_ S. Hannon, and S. Henderson, 1998: Coherent Doppler lidar measurements of wind field statistics. Bound.-Layer Meteor., 86, 233-256.

Gal-Chen, T., M. Xu, and W. L. Eberhard, 1992: Estimation of atmospheric boundary layer fluxes and other turbulence parameters from Doppler lidar data. J. Geophys. Res., 97, 18 409-18 423.

Harrison, R. G., A. M. Heath, R. J. Hogan, and G. W. Rogers, 2009: Comparison of balloon-carried atmospheric motion sensors with Doppler lidar turbulence measurements. Rev. Sci. Instrum., 80, 026108.

Hignett, P., 1991: Observations of diurnal variation in a cloudcapped marine boundary layer. J. Atmos. Sci., 48, 1474-1482.

Hill, M. K., B. J. Brooks, S. J. Norris, M. H. Smith, I. M. Brooks, and G. de Leeuw, 2008: A Compact Lightweight Aerosol Spectrometer Probe (CLASP). J. Atmos. Oceanic Technol., 25, 1996-2006.

Kolmogorov, A. N., 1941: Dissipation of energy in locally isotropic turbulence. Dokl. Akad. Nauk SSSR, 32, 16-18.

Lenschow, D. H., V. Wulfmeyer, and C. Senff, 2000: Measuring second- through fourth-order moments in noisy data. $J$. Atmos. Oceanic Technol., 17, 1330-1347.

Lothon, M., D. H. Lenschow, and S. D. Mayor, 2009: Doppler lidar measurements of vertical velocity spectra in the convective planetary boundary layer. Bound.-Layer Meteor., 132, 205-226.

Martin, D., and Coauthors, 2009: Tracer concentration profiles measured in central London as part of the REPARTEE campaign. Atmos. Chem. Phys. Discuss., 9, 25 245-25 274.

McKay, J. A., 1998: Modeling of direct detection Doppler wind lidar. I. The edge technique. Appl. Opt., 37, 6480-6486. 
O’Connor, E. J., R. J. Hogan, and A. J. Illingworth, 2005: Retrieving stratocumulus drizzle parameters using Doppler radar and lidar. J. Appl. Meteor., 44, 14-27.

Panagi, P., E. Dicks, G. Hamer, and J. Nash, 2001: Preliminary results of the routine comparison of wind profiler data with the Meteorological Office Unified Model vertical wind profiles. Phys. Chem. Earth, 26B, 187-191.

Paquin, J. E., and S. Pond, 1971: The determination of the Kolmogoroff constants for velocity, temperature and moisture from second and third order structure functions. J. Fluid Mech., 50, 257-269.

Pearson, G., F. Davies, and C. Collier, 2009: An analysis of the performance of the UFAM pulsed Doppler lidar for observing the boundary layer. J. Atmos. Oceanic Technol, 26, 240-250.

Pinsky, M., A. Khain, and H. Krugliak, 2008: Collisions of cloud droplets in a turbulent flow. Part V: Application of detailed tables of turbulent collision rate enhancement to simulation of droplet spectra evolution. J. Atmos. Sci., 65, 357-374.
Rye, B. J., 1979: Antenna parameters for incoherent backscatter heterodyne lidar. Appl. Opt., 18, 1390-1398.

—_, and R. M. Hardesty, 1993: Discrete spectral peak estimation in incoherent backscatter heterodyne lidar. I: Spectral accumulation and the Cramer-Rao lower bound. IEEE Trans. Geosci. Remote Sens., 31, 16-27.

Siebert, H., M. Wendisch, T. Conrath, U. Teichmann, and J. Heintzenberg, 2003: A new tethered balloon-borne payload for fine-scale observations in the cloudy boundary layer. Bound.-Layer Meteor., 106, 461-482.

_ K. Lehmann, and M. Wendisch, 2006: Observations of smallscale turbulence and energy dissipation rates in the cloudy boundary layer. J. Atmos. Sci., 63, 1451-1466.

Taylor, G. I., 1935: Statistical theory of turbulence. Roy. Soc. London Proc., 151A, 421-444.

Wehner, B., and Coauthors, 2010: Observations of turbulenceinduced new particle formation in the residual layer. Atmos. Chem. Phys., 10, 4319-4330. 